\title{
BMJ Open Time-driven activity-based costing of total knee replacements in Karachi, Pakistan
}

\author{
Rabia Mansoor Khan, ${ }^{1}$ Katherine Albutt, ${ }^{\oplus 2,3}$ Muhammad Abdullah Qureshi, ${ }^{1}$ \\ Zara Ansari, ${ }^{4}$ Gustaf Drevin, ${ }^{2,5}$ Swagoto Mukhopadhyay, ${ }^{2,6}$ Mansoor Ali Khan, ${ }^{7}$ \\ Muhammad Amin Chinoy, ${ }^{7}$ John Meara, ${ }^{2}$ Hamidah Hussain ${ }^{8}$
}

To cite: Khan RM, Albutt K, Qureshi MA, et al. Timedriven activity-based costing of total knee replacements in Karachi, Pakistan. BMJ Open 2019;0:e025258. doi:10.1136/ bmjopen-2018-025258

- Prepublication history and additional material for this paper are available online. To view these files, please visit the journal online (http://dx.doi. org/10.1136/bmjopen-2018025258).

Received 13 July 2018 Revised 5 April 2019 Accepted 8 April 2019
Check for updates

(C) Author(s) (or their employer(s)) 2019. Re-use permitted under CC BY-NC. No commercial re-use. See rights and permissions. Published by BMJ.

For numbered affiliations see end of article.

Correspondence to Rabia Mansoor Khan; rabiamansoork@gmail.com

\section{ABSTRACT}

Introduction Osteoarthritis of the knee has been identified as the most common disability in Pakistan. Total knee replacement (TKR) surgery is the curative treatment for advanced osteoarthritis of the knee; however, cost remains one of the barriers to effective and timely service delivery.

Objective We conducted a time-driven activity-based costing (TDABC) analysis of TKR to identify major cost drivers and areas for process improvement.

Methods and analysis We performed a prospective TDABC analysis of patients who underwent bilateral TKR at The Indus Hospital (TIH) during a 14-month period from October 2015 to December 2016. Detailed process maps were developed for each phase of the care cycle. Time durations and costs were allocated to each resource utilised and aggregated across the care cycle, including personnel, direct and indirect costs.

Results We identified seven care phases for a complete TKR care cycle and created their detailed process maps. Major time contributors were ward stay and discharge (20160 min), TKR surgery (563 min) and surgical admission (333 min). Overall, $92.10 \%$ of time is spent during the ward stay and discharge phase of care. Patients remain hospitalised for an average of 14 days postoperatively. Overall institutional cost of a TKR at TIH was US\$4360.51 (Pakistani rupees 456 981.17) per bilateral TKR surgery. The overall primary cost drivers for the full bundle of care were consumables used during TKR surgery itself, consumables utilised in the wards and personnel costs contributing $57.64 \%, 27.45 \%$ and $12.03 \%$ of total costs, respectively.

Conclusion Utilising TDABC allowed us to obtain a granular analysis of time and cost that was subsequently used to inform quality process improvement initiatives. In low-resource settings, such as Pakistan, TDABC has the potential to be a useful tool to guide resource allocation and process improvement.

\section{BACKGROUND}

The growing burden of non-communicable and age-related disease in low/ middle-income countries (LMICs) poses fundamental challenges to already resource-strained health systems. Between 1990 and 2013, the

\section{Strengths and limitations of this study}

- Time-driven activity-based costing (TDABC) analysis is an effective and accurate methodology to identify major cost drivers and areas for process improvement.

- In low-resource settings, such as Pakistan, TDABC has the potential to be a useful tool to allocate resources in the most effective manner with minimum wastage.

- The study demonstrates the utility of a thorough micro-costing methodology that can be scaled and adapted to other low-resource settings.

- Process maps are resource-intensive to develop and reflect a fluid process that is constantly in flux.

- Given the free care model at TIH, certain capital and overhead costs were not readily accessible requiring several cost assumptions to be made.

prevalence of osteoarthritis has quadrupled in LMICs. ${ }^{1}$ Osteoarthritis of the knee has been identified as the most common disability in Pakistan with a prevalence of 29.7-37.0 per 1000 people. ${ }^{2}$ Pain and impaired mobility as a result of osteoarthritis often results in considerable reduction of quality of life. For patients in early stages of osteoarthritis, unicompartmental knee replacement ${ }^{3}$ could be an option. However, total knee replacement (TKR) surgery is the curative treatment for advanced osteoarthritis of the knee and standard of care around the globe. Patients presenting at The Indus Hospital are mostly categorised with Kellgren and Lawrence ${ }^{4}$ grade 4 condition with severe deformity of the knee which often necessitates a TKR. In resource-constrained environments, health facilities and providers face significant financial challenges in meeting the demand for TKR surgery.

Cost is one of many barriers to effective and timely service delivery in LMICs for conditions such as knee osteoarthritis. Timedriven activity-based costing (TDABC) is a 
value-based costing methodology that is applicable to health systems in LMICs, ${ }^{5}$ as it allows system inefficiencies to be reduced, efficiencies to be maximised and cost containment to be evaluated based on the time required to perform the service and unit cost of supplying capacity. The methodology involves: (1) defining the care cycle; (2) mapping the processes involved; (3) determining the time spent utilising each resource; (4) estimating the direct and indirect costs for each resource; (5) multiplying cost per resource by the time spent to determine the capacity cost rate (CCR) and (6) aggregating all costs. ${ }^{6}$ Importantly, this costing approach is generalisable across a range of medical conditions and clinical contexts. ${ }^{7}$ The 'bottom-up' approach allows researchers, clinicians and policymakers to estimate the clinical and administrative cost of the full life cycle of the medical condition, thereby informing process improvement and cost containment. ${ }^{89}$

The Indus Hospital (TIH) in Karachi, Pakistan, is a 273-bed free-of-charge hospital that provides free quality medical and surgical care to patients, including TKR. Despite being a tertiary facility that could handle higher volumes, TIH has been forced to limit the number of TKRs due to high costs. Consequently, the current waiting time for TKR at TIH averages approximately 5 years. The application of cost-containment and process improvement strategies was thought to be necessary to enhance TIH's TKR efficiency and throughput. TDABC has effectively estimated cost and efficiency of TKR surgery in multiple high-income settings; however, this study is the first to describe the application of TDABC for knee replacement in an LMIC. ${ }^{9-11}$ We conducted a TDABC analysis of TKR to identify major cost drivers and areas for process improvement at TIH in Karachi, Pakistan.

\section{METHODS}

We performed a prospective TDABC analysis of patients who underwent bilateral TKR at TIH during a 14-month period from October 2015 to December 2016.

\section{The care cycle}

TDABC is based on the unit cost of supplying time and capacity required to perform the service in question. The care cycle was defined as the time from the patient's initial appointment at the family medicine clinic (FMC), a general practice clinic for the hospital that serves as a patient's first point of contact, until their 3-month postoperative visit. From the FMC, patients are typically referred to the orthopaedic department for a specialist consultation, which includes radiologic imaging and preoperative anaesthesia assessment. If patients require surgery, their names are added to the waiting list. Typically, patients are admitted a day prior to the surgery for preoperative reassessment and preparation. On the day of surgery, after a short wait in the holding area, patients enter the operating theatre. For bilateral knee surgery, the TKRs are performed by two surgeons simultaneously. The postoperative admission period involves physiotherapy in the ward until discharge. Postoperative follow-up is conducted at the orthopaedic consultation clinic at 15, 30 and 90 days after discharge.

\section{Process mapping}

Detailed process maps were developed for each phase of the care cycle to demonstrate and understand all steps, resources and decisions in a patient care cycle. Such process mapping depicts the activities performed for patients as they transit through the cycle of care, incorporating capacity-supplying resources they encounter such as personnel, facilities, equipment and consumables. Iterative discussions were held with key stakeholders to understand the clinical workflow for TKR patients at TIH. For each activity in the process map, between five to eight patients were directly observed by the research team and followed as they progressed through the complete care cycle. Activities related to the process were timed and recorded. Process or activity times were defined as the time spent receiving care or while a staff member is directly involved in facilitating or assisting a patient, whereas wait times were defined as the time spent waiting to receive care. Data on staffing numbers, resources and time duration of activities were collected by direct observation until stable averages were reached. Subsequently, care maps were validated through multidisciplinary meetings with frontline staff and management.

\section{Personnel costs}

The cost per unit time for all personnel directly involved in TKR patient care (doctors, nurses, receptionists, welfare officers, phlebotomists and so on) was tabulated using salary figures from human resources department. Total working hours (excluding holidays, leaves and breaks) were obtained from duty rosters. The time spent at each point of contact was directly obtained through process mapping. We then calculated salary costs per minute based on CCR for all personnel who participated in the care of TKR patients. Staff cost per minute was multiplied by the number of minutes spent with each patient.

\section{Direct costs}

Consumable costs of implants, medications, testing and supplies were estimated at TIH's discounted purchase. Direct and support costs for each resource were collected from the finance, maintenance, supply chain and central stores departments at TIH.

\section{Indirect costs}

Costs that do not directly contribute to individual patient care and yet contribute to overall cost were included as indirect costs. Costs such as salary of hospital administrators, health systems information technology, operating theatre maintenance, utilities and departmental overheads were incorporated in the analysis. Cost data for space and electricity consumption were calculated by direct consumption for each process point. Overhead costs were obtained from the finance department at TIH and were allocated for each section of the hospital based 


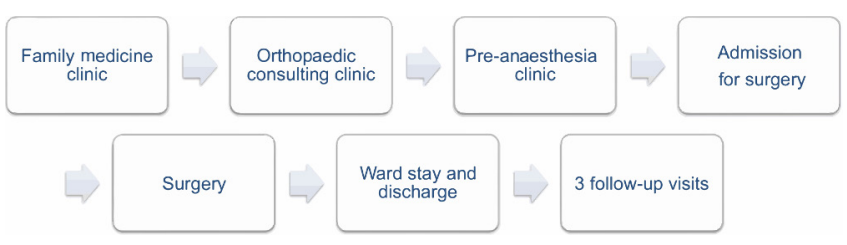

Figure 1 Summary of patient pathway for bilateral total knee replacement (TKR). The process pathway identified seven unique points visited by a bilateral TKR patient which were family medicine clinic, orthopaedic consulting clinic, preanaesthesia clinic, admission for surgery, TKR surgery, ward stay and discharge, and follow-up.

on cost of equipment, payroll, volume of patients, area per square foot and number of employees. Depreciation was calculated for buildings and machinery.

\section{Data analysis}

Descriptive statistics were used to report minutes spent on processes and activities. Costing was performed using Pakistani rupees (PKR) and converted to US dollars (US\$) at an average exchange rate for the duration of the study (PKR 104.8 to US\$1.00) using State Bank of Pakistan's historical currency conversion rates for the study period. All costs were aggregated across the care cycle.

\section{Ethics}

All personnel and patient information was anonymised. Ethical clearance for the research was granted by the Interactive Research and Development Institutional Review Board and Boston Children's Hospital that waived the requirement of informed consent as it was no more than a minimal risk study. Patient processes were observed only for time information, and hospital authorities were informed.

\section{Patient and public involvement}

There was no direct patient involvement. Instead, this was a prospective observational study of the time a TKR patient spent visiting different phases of care at TIH.
RESULTS

The TKR pathway was prospectively mapped on 55 TKR patients. The average age for patients who had their TKRs during the study duration was $58.4 \pm 1.7$ years and the male to female distribution was $31 \%$ and $69 \%$, respectively. Median length of stay (LOS) was 14 days (IQR 6) and all patients were discharged to their own home.

\section{Phases of care}

We identified seven phases of care for a complete TKR care cycle. The phases in order from admission to discharge were: FMC, orthopaedic consulting clinic, preanaesthesia clinic, admission for surgery, TKR surgery, ward stay and discharge, and follow-up (figure 1). Detailed process maps for each phase of care were created (figure 2 contains a sample process map; remaining process maps are included in the online supplementary material). A total of 28 types of personnel were involved in the TKR pathway. All clinical care activities were mapped in detail during a patient's entire hospital course. Time duration and cost were allocated to each resource utilised (figure 3).

\section{Process times}

Major time contributors were ward stay and discharge (20160 min), TKR surgery: its set-up, procedure and winding up from the theatre (563 min), and surgical admission (333 min) (table 1). Overall, 92.10\% of time is spent during the ward stay and discharge phase of care. Patients remain hospitalised for an average of 14 days postoperatively. At all points of contact, the patient spent more than three-quarters of their time waiting between processes, relative to overall process times, with the exception being the TKR surgery itself. The longest wait times were observed for admission processes, whereas the operating theatre process used time most efficiently.

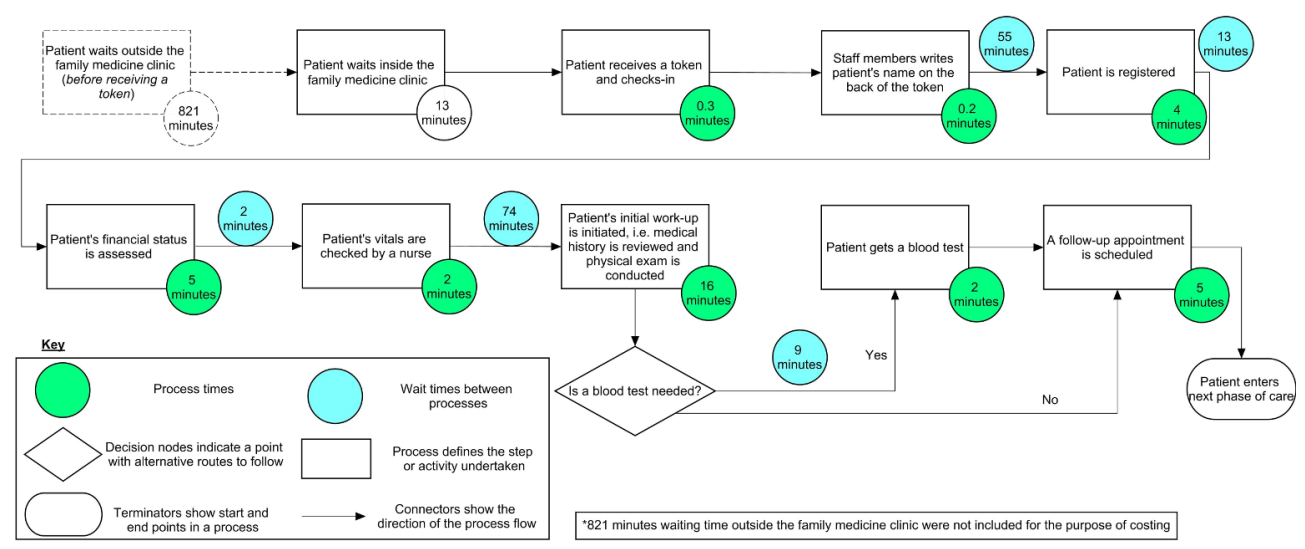

Figure 2 Process map for a visit to the family medicine clinic. Process map for the first point of contact at the hospital for a total knee replacement patient is shown. Rectangles denote different activities, and circles denote the duration they take. Green circles denote the time taken for a process, whereas blue circles denote the time taken waiting between processes. Rhombus denotes a decision node with alternative routes to follow (for example, 'is a blood test needed?'). Stadium shape denotes the end point in a phase of care (patient enters next phase of care). Connectors show the direction of the process flow. 


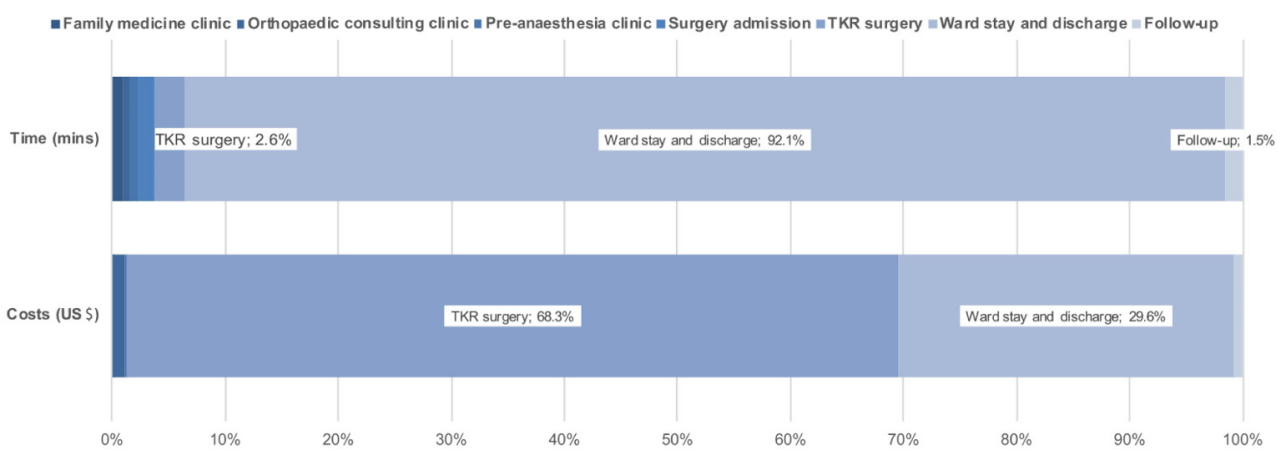

Figure 3 Distribution of time and costs by segment of care. Top percentage bar diagram denotes the percentage allocation of time (minutes) for the entire length of process times spent by a total knee replacement (TKR) patient. Bottom percentage bar diagram denotes the percentage allocation of costs (US\$) for the total cost spent on a TKR patient. Horizontal axis shows the percentage (from 0 to 100) dedicated to time and costs, respectively, for the two percentage bar diagrams.

\section{Overall cost}

Overall institutional cost of a TKR at TIH was US $\$ 4360.51$ (PKR 456 981.17) per bilateral TKR surgery. The cost for personnel in the TKR care cycle was US $\$ 524.44$ (table 2 ). The total cost of consumables was US $\$ 3737.49$ of which US $\$ 2513.55$ were incurred during the TKR surgical procedure (table 3$)$. More than half of total cost $(57.64 \%$; US \$2513.55) is attributable to knee implants, anaesthesia, and consumables used during the procedure. Overhead costs added up to US $\$ 98.58$, representing only $2.26 \%$ of total cost (table 1).

The overall primary cost drivers for the full bundle of care were consumables used during TKR surgery itself, consumables utilised in the wards and personnel costs contributing $57.64 \%, 27.45 \%$ and $12.03 \%$ of total costs, respectively. Overall, the TKR surgery phase accounts for $68.28 \%$ of overall cost, with ward stay and discharge contributing $29.58 \%$ and the remaining five phases contributing less than $1 \%$ of total cost each.

\section{DISCUSSION}

Utilising TDABC, we calculated the total cost of a bilateral TKR surgery from initial consultation through 3-month postoperative follow-up at TIH to be US $\$ 4360.51$. The major cost drivers identified were consumables used during TKR surgery (primarily implants), consumables utilised in the wards (including items and services such as IV and pain medications, physiotherapy, rehabilitation, meals and bed occupancy charges) and personnel costs. Concurrently, the process mapping identified possible interventions for quality improvement in the TKR pathway.

The earliest costing study published for knee arthroplasty was performed over three decades ago and estimated the total cost of inpatient treatment at approximately US $\$ 5490 .^{12}$ More recent studies have estimated costs at US $\$ 8500-$ US $\$ 17000$, but few of these studies have detailed their costing methodology or taken into account indirect costs. ${ }^{13-16}$ A TDABC analysis of TKR

Table 1 Cost and duration by phase of care

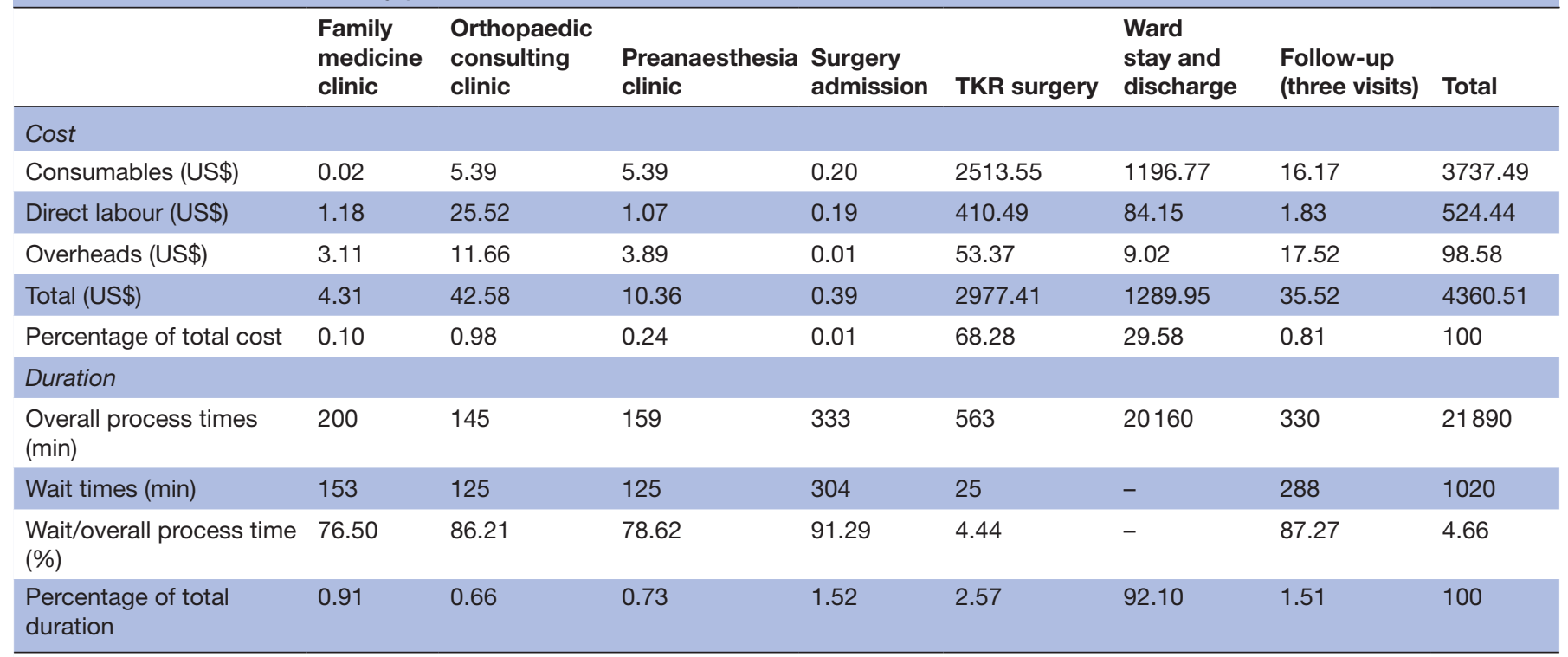

TKR, total knee replacement. 


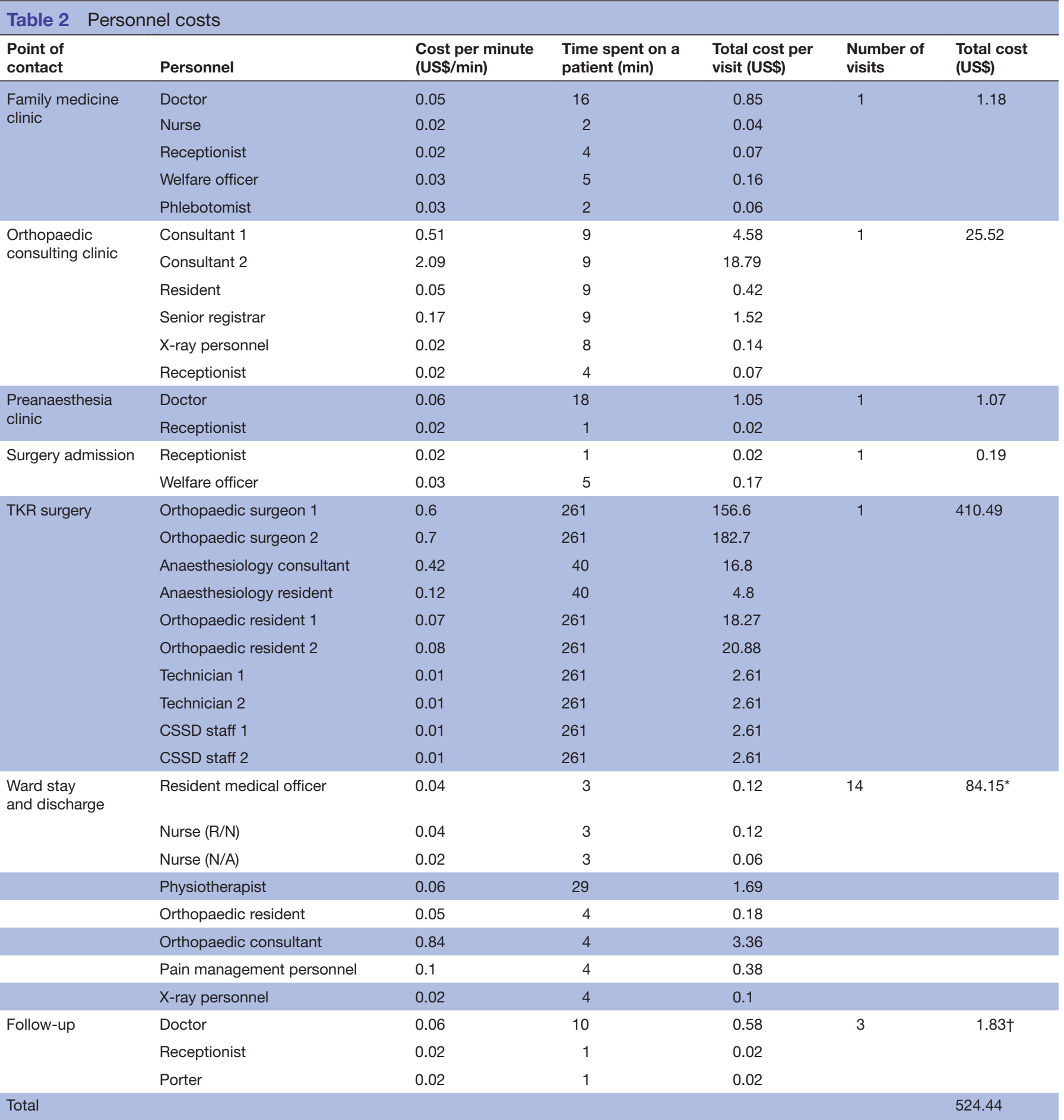

${ }^{*}$ For a total of 14 days.

†For a total of three visits.

CSSD, central sterile services department; TKR, total knee replacement.

surgery at a London teaching hospital in 2015 reported a cost of approximately US $\$ 7696 .{ }^{11}$ That same year in the USA, the cost of TKR surgery using TDABC was reported as US $\$ 13661 .{ }^{17}$ Notably, the cost of bilateral TKR surgery at TIH (US\$4360) is approximately one third of the cost of unilateral TKR in the USA when utilising the TDABC methodology in both settings.
At TIH, more than two-thirds of the overall cost is attributable to the TKR surgery itself, with implants and consumables driving this cost. Knee implants, a major cost driver for TKR around the world, has been a focus of research and discussion within the orthopaedic community. ${ }^{18} 19$ Notably, the cost of implants was calculated at the preferential rate provided by vendors to 
Table 3 Breakdown of consumable costs

\begin{tabular}{|c|c|c|c|c|}
\hline Consumable & Point of utilisation & Cost (US\$) & $\begin{array}{l}\% \text { of } \\
\text { Consumable cost }\end{array}$ & $\%$ of Overall cost \\
\hline Implants & TKR surgery & 1720.88 & 46.04 & 39.47 \\
\hline $\begin{array}{l}\text { Operating theatre and } \\
\text { anaesthesia charges }\end{array}$ & TKR surgery & 448.78 & 12.01 & 10.29 \\
\hline Ward consumables & Ward stay and discharge & 698.42 & 18.69 & 16.02 \\
\hline Bandages & Ward stay and discharge & 238.39 & 6.39 & 5.48 \\
\hline X-rays (eight films) & $\begin{array}{l}\text { Orthopaedic consulting clinic, } \\
\text { preanaesthesia clinic, ward stay and } \\
\text { discharge (3), follow-up (3) }\end{array}$ & 42.84 & 1.15 & 0.98 \\
\hline
\end{tabular}

TKR, total knee replacement.

$\mathrm{TIH}$ and not the market rate, suggesting that implants may be an even larger cost driver. This reinforces the importance of central negotiation with suppliers for discounting and cost reduction, particularly in the not-for-profit sector.

Ward stay, primarily driven by the average LOS of 14 days, amounts to nearly one third of the overall cost. This is in stark contrast to high-income countries, where LOS averages 2 days. $^{10}{ }^{20}$ As many TIH patients are from lower socioeconomic households and often travel long distances to reach the facility, concerns regarding postoperative surveillance and rehabilitation have led to prolonged postoperative admissions, contributing significantly to overall cost. Given the influence of LOS on overall cost, a number of studies in high-income settings have explored demonstrating how enhanced recovery programmes (ERP) can reduce LOS without increasing readmission or complication rates. ${ }^{21-23}$ Each additional day at TIH costs US\$92.1, suggesting that this is a ripe domain for process improvement and cost containment.

Using process maps, one can obtain granular analysis of time and cost that can be used to inform quality process improvement initiatives. Transparent cost analysis and process maps allow cost reduction and process redesign to improve outcomes at similar or lower costs. As a result of our study, numerous improvement opportunities were identified by management and clinicians, including (1) reduction in overall LOS through the adoption of an educational ERP; (2)addressing long wait times; (3) cost of multiple surgeons in theatre; (4) revisiting and revising hospital finances at the departmental level and (5) application of TDABC to other hospital processes. These opportunities and proposed solutions are outlined in detail (table 4).
Our study has several inherent limitations. First, process maps are resource intensive to create and reflect a fluid process that is constantly in flux. Second, given the free care model at TIH, certain capital and overhead costs were not readily accessible requiring several cost assumptions to be made. Equipment and consumables that were donated, for example, were included in the costing at market rates. Third, the estimate of TKR cost generated is perhaps impacted by financing models and procurement methods that are unique to TIH. Despite this, our study demonstrates the utility of a thorough microcosting methodology that can be scaled and adapted to other low-resource settings.

Due to ever increasing cost of medical care, the provision of high-quality value-based healthcare is a challenge for medical institutions across the globe, in both high-income settings and LMICs alike. In a low-resource setting, TDABC has the potential to be a useful tool to guide resource allocation and process improvement. ${ }^{5} 17$ Directly tracing the patient flow allowed inductive modelling of the existing system of care and generation of discussion about the optimisation of the care cycle to create better outcomes and efficiencies. Such fine-tuning of care processes is particularly important in low-resourced health systems. ${ }^{5}$

\section{CONCLUSIONS}

In this manuscript we explain the development, the relevance and the impact of TDABC in a low-resource setting. The success of applying these methods to a small TKR patients' cohort in this setting highlights the potential for studies with more heterogeneous groups of patients to better inform policy and programming. Reduction in wait times, appropriate discounting of implant costs, 
Table 4 Improvement opportunities

\begin{tabular}{|c|c|c|c|}
\hline Improvement opportunity & Targeted phase of care & Process issue & Plan of action \\
\hline Overall LOS & Ward stay and discharge & Cost and time & $\begin{array}{l}\text { Adoption of educational ERP } \\
\text { to reduce LOS }\end{array}$ \\
\hline Hospital financing systems & All & Cost & $\begin{array}{l}\text { Revisiting and revising hospital } \\
\text { finances at departmental level } \\
\text { based on lessons learnt from } \\
\text { TDABC }\end{array}$ \\
\hline
\end{tabular}

ERP, enhanced recovery programmes; LOS, length of stay; TDABC, time-driven activity-based costing; TKR, total knee replacement.

reduction in LOS by adopting an ERP and other process improvement initiatives are proposed strategies for reducing the overall treatment cost.

\section{Author affiliations}

${ }^{1}$ Indus Hospital Research Center, The Indus Hospital, Karachi, Pakistan

${ }^{2}$ Program in Global Surgery and Social Change, Harvard Medical School, Boston, Massachusetts, USA

${ }^{3}$ General Surgery, Massachusetts General Hospital, Boston, Massachusetts, USA ${ }^{4}$ Global Health Directorate, Indus Health Network, Karachi, Pakistan

${ }^{5}$ Department for Public Health Sciences, Karolinska Institutet, Stockholm, Sweden ${ }^{6}$ Department of Surgery, University of Connecticut, Farmington, Connecticut, USA ${ }^{7}$ Department of Orthopedics, The Indus Hospital, Karachi, Pakistan

${ }^{8}$ Global Health, Interactive Research and Development (IRD), Global, Singapore

Acknowledgements The authors thank and acknowledge Irfan Nasir, Asim Yar, Niaz Muhammad, Muhammad Asif and Waqar Ahmed for their help in collecting and compiling data for the study.

Contributors Conception: HH, MAC, MAK, RMK, ZA, SM and JM. Data collection: RMK, MAQ and ZA. Analysis: RMK, MAQ, ZA, HH and KA. Writing: KA, GD, HH, RMK, $M A Q$ and JM.

Funding This project was made possible through contributions from the Harvard Medical School Center for Global Health Delivery-Dubai, and the Program in Global Surgery and Social Change at Harvard Medical School. GD was supported by the Swedish Society of Medicine and the Erik and Göran Ennerfelt Foundation.

Competing interests None declared.

Patient consent for publication Not required.

Ethics approval This study was approved by the institutional review boards at Boston Children's Hospital and Institutional Review Board—Interactive Research and Development.

Provenance and peer review Not commissioned; externally peer reviewed.

Data sharing statement No data are available. Costing data were collected by the research team which are confidential.

Open access This is an open access article distributed in accordance with the Creative Commons Attribution Non Commercial (CC BY-NC 4.0) license, which permits others to distribute, remix, adapt, build upon this work non-commercially, and license their derivative works on different terms, provided the original work is properly cited, appropriate credit is given, any changes made indicated, and the use is non-commercial. See: http://creativecommons.org/licenses/by-nc/4.0/.

\section{REFERENCES}

1. Moradi-Lakeh M, Forouzanfar MH, Vollset SE, et al. Burden of musculoskeletal disorders in the Eastern Mediterranean Region, 1990-2013: findings from the Global Burden of Disease Study 2013. Ann Rheum Dis 2017;76:1365-73.

2. Farooqi A, Gibson T. Prevalence of the major rheumatic disorders in the adult population of north Pakistan. Br J Rheumatol 1998;37:491-5.

3. Burn E, Liddle AD, Hamilton TW, et al. Cost-effectiveness of unicompartmental compared with total knee replacement: a population-based study using data from the National Joint Registry for England and Wales. BMJ Open 2018;8:e020977.

4. Kohn MD, Sassoon AA, Fernando ND. Classifications in brief: Kellgren-Lawrence Classification of Osteoarthritis. Clin Orthop Relat Res 2016;474:1886-93.

5. McBain RK, Jerome G, Warsh J, et al. Rethinking the cost of healthcare in low-resource settings: the value of time-driven activitybased costing. BMJ Glob Health 2016;1:e000134.

6. Kaplan RS, Anderson SR. Time-driven activity-based costing. Harv Bus Rev 2004;82:131-8.

7. Yu YR, Abbas PI, Smith CM, et al. Time-driven activity-based costing to identify opportunities for cost reduction in pediatric appendectomy. J Pediatr Surg 2016;51:1962-6.

8. Daniels KM, Lappi MD, Sporn SF, et al. Assessing the cost of prophylactic antibiotic use after cleft lip and lip adhesion Procedures. $J$ Healthc Manag 2016;61:282-9.

9. Keel G, Savage C, Rafiq M, et al. Time-driven activity-based costing in health care: a systematic review of the literature. Health Policy 2017;121:755-63.

10. Andreasen SE, Holm HB, Jørgensen M, et al. Time-driven activity-based cost of fast-track total hip and knee arthroplasty. $J$ Arthroplasty 2017;32:1747-55.

11. Chen A, Sabharwal S, Akhtar K, et al. Time-driven activity based costing of total knee replacement surgery at a London teaching hospital. Knee 2015;22:640-5.

12. Dreghorn CR, Roughneen P, Graham J, et al. The real cost of joint replacement. Br Med J 1986;292:1636-7.

13. Effenberger H, Rehart S, Zumstein MD, et al. Financing in knee arthroplasty: a benchmarking analysis. Arch Orthop Trauma Surg 2008;128:1349-56. 
14. Ilfeld BM, Mariano ER, Williams BA, et al. Hospitalization costs of total knee arthroplasty with a continuous femoral nerve block provided only in the hospital versus on an ambulatory basis: a retrospective, case-control, cost-minimization analysis. Reg Anesth Pain Med 2007;32:46-54.

15. Palsis JA, Brehmer TS, Pellegrini VD, et al. The cost of joint replacement: comparing two approaches to evaluating costs of total hip and knee arthroplasty. J Bone Joint Surg Am 2018;100:326-33.

16. Zhang $\mathrm{Y}$, Zhang $\mathrm{H}$, Clarke $\mathrm{HD}$, et al. Analysis of total joint arthroplasty costs in Chinese patients. J Arthroplasty 2012;27:1423-8.

17. Akhavan S, Ward L, Bozic KJ. Time-driven activity-based costing more accurately reflects costs in arthroplasty surgery. Clin Orthop Relat Res 2016;474:8-15.

18. Jain NB. Variability in costs associated with total hip and knee replacement implants. J Bone Joint Surg Am 2012;94:e140.
19. Robinson JC, Pozen A, Tseng S, et al. Variability in costs associated with total hip and knee replacement implants. J Bone Joint Surg Am 2012;94:1693-8.

20. Solomon L, Sullivan T, Ding G. Length of hospital stay after total knee replacement: the effect of patient controled Analgesia. Orthopaedic Proceedings 2012.

21. Dwyer AJ, Thomas W, Humphry S, et al. Enhanced recovery programme for total knee replacement to reduce the length of hospital stay. J Orthop Surg 2014;22:150-4.

22. McDonald DA, Siegmeth R, Deakin AH, et al. An enhanced recovery programme for primary total knee arthroplasty in the United Kingdom--follow up at one year. Knee 2012;19:525-9.

23. Paton F, Chambers D, Wilson P, et al. Effectiveness and implementation of enhanced recovery after surgery programmes: a rapid evidence synthesis. BMJ Open 2014;4:e005015. 